\title{
TUNNEL NUMBER ONE KNOTS, m-SMALL KNOTS AND THE MORIMOTO CONJECTURE
}

\author{
GUOQIU YANG, XUNBO YIN, AND FENGCHUN LEI
}

(Communicated by Daniel Ruberman)

\begin{abstract}
In the present paper, we show that the Morimoto Conjecture on the super additivity of the tunnel numbers of knots in $S^{3}$ is true for knots $K_{1}, K_{2}$ in $S^{3}$ in which each $K_{i}$ is either a tunnel number one or $m$-small, $i=1,2$. This extends two known results by Morimoto.
\end{abstract}

\section{INTRODUCTION}

For a knot $K$ in $S^{3}$, let $N(K)$ be a regular neighborhood of $K$ in $S^{3}$ and $E(K)=\overline{S^{3}-N(K)}$, the complement of $K$. An unknotting tunnel system for $K$ is a collection $\Gamma=\left\{\gamma_{1}, \cdots, \gamma_{t}\right\}$ of pairwise disjoint simple arcs properly embedded in $E(K)$ such that $\overline{E(K)-N(\Gamma)}$ is a handlebody, where $N(\Gamma)$ is a regular neighborhood of $\Gamma$ in $E(K)$. Let $t(K)=\min \{|\Gamma|: \Gamma$ is an unknotting tunnel system for $K\}$, and call $t(K)$ the tunnel number of $K$. Clearly, an unknotting tunnel system $\Gamma=\left\{\gamma_{1}, \cdots, \gamma_{t}\right\}$ for $K$ gives rise to a Heegaard splitting $V \cup_{S} W$ for $E(K)$ with $g(S)=t+1$, where $V$ is the closure of a regular neighborhood of $(\partial E(K)) \cup \gamma_{1} \cup \cdots \cup \gamma_{t}$ in $E(K), W=\overline{E(K)-V}$, and vice versa. Thus, the Heegaard genus $g(E(K))$ of $E(K)$ is $t(K)+1$.

Given two knots $K_{1}, K_{2} \subset S^{3}$, it is a natural question to ask: How does the sum of $t\left(K_{1}\right)$ and $t\left(K_{2}\right)$ compare with the tunnel number $t\left(K_{1} \# K_{2}\right)$ of the connected sum $K_{1} \# K_{2}$ of $K_{1}$ and $K_{2}$ ? It is not hard to check that $t\left(K_{1} \# K_{2}\right) \leq t\left(K_{1}\right)+$ $t\left(K_{2}\right)+1$ always holds. Hence there are three possibilities for $K_{1} \# K_{2}$ : (1) Super additive: $t\left(K_{1} \# K_{2}\right)=t\left(K_{1}\right)+t\left(K_{2}\right)+1 ;(2)$ Additive: $t\left(K_{1} \# K_{2}\right)=t\left(K_{1}\right)+t\left(K_{2}\right)$; (3) Sub-additive: $t\left(K_{1} \# K_{2}\right) \leq t\left(K_{1}\right)+t\left(K_{2}\right)-1$.

Morimoto ([12, [13]) found infinitely many pairs of knots $K_{1}, K_{2} \subset S^{3}$ such that $t\left(K_{1} \# K_{2}\right) \leq t\left(K_{1}\right)+t\left(K_{2}\right)-1$, and Kobayashi (2]) generalized the results by showing that for any positive integer $m$, there exist knots $K_{1}, K_{2} \subset S^{3}$ so that $t\left(K_{1} \# K_{2}\right) \leq t\left(K_{1}\right)+t\left(K_{2}\right)-m$. On the other hand, it is well known that there exist infinitely many examples of $K_{1}, K_{2} \subset S^{3}$ with $t\left(K_{1} \# K_{2}\right)=t\left(K_{1}\right)+t\left(K_{2}\right)$. Moreover, Moriah-Rubinstein ([9]) and Morimoto-Sakuma-Yokota ([15]) showed that

Received by the editors July 15, 2011 and, in revised form, November 8, 2011; December 20, 2011; January 18, 2012; and February 8, 2012.

2010 Mathematics Subject Classification. Primary 57M99.

Key words and phrases. Tunnel number, $m$-small, Morimoto Conjecture.

The first author was supported in part by two grants of NSFC (No. 11001065 and No. 11071106) and by two grants of HITQNJS (No. 2009.029 and No. 20100471066).

The second author was supported in part by a grant of NSFC (No. 11001065).

The third author was supported in part by a key grant of NSFC (No. 10931005). 
there exist infinitely many pairs of knots $K_{1}, K_{2} \subset S^{3}$ satisfying $t\left(K_{1} \# K_{2}\right)=$ $t\left(K_{1}\right)+t\left(K_{2}\right)+1$, so the bound is best possible. Refer to the survey paper [8] for more details. It is an interesting question to ask as to what kinds of knots have the super additivity of tunnel numbers.

A Heegaard splitting $V \cup_{S} W$ of $E(K)$ is primitive if there exists a spanning annulus $A$ in $V$ with $\partial A=\alpha \cup \gamma, \alpha \subset \partial_{-} V=\partial E(K)$ is a meridian of $K$ and $\gamma \subset S$ meets an essential disk of $W$ in one point. A knot $K$ in $S^{3}$ is $\mu$-primitive if $E(K)$ has a minimal primitive Heegaard splitting.

The results mentioned above lead to the following conjecture by Morimoto ([12] or $[8])$ :

Conjecture 1.1. Let $K_{1}, K_{2}$ be knots in $S^{3}$. Then $t\left(K_{1} \# K_{2}\right)=t\left(K_{1}\right)+t\left(K_{2}\right)+1$ if and only if neither $K_{1}$ nor $K_{2}$ is $\mu$-primitive.

Conjecture 1.1 is known to be true for tunnel number one knots in $S^{3}$ by Morimoto ([1] $)$ :

Theorem 1.2. Let $K_{1}, K_{2}$ be two tunnel number one knots in $S^{3}$. Then $t\left(K_{1} \# K_{2}\right)$ $=t\left(K_{1}\right)+t\left(K_{2}\right)+1$ if and only if neither $K_{1}$ nor $K_{2}$ is $\mu$-primitive.

A surface $F$ properly embedded in $E(K)$ is meridional if $\partial F \neq \emptyset$ and each component of $\partial F$ is a meridian of $K$. A knot $K$ is meridionally small or $m$-small if there is no meridional essential surface in $E(K)$. A knot $K$ is small if $E(K)$ contains no closed essential surface.

Morimoto later ([12]) showed that Conjecture 1.1 is true for two prime knots in $S^{3}$ which are $m$-small:

Theorem 1.3. Let $K_{1}, K_{2}$ be two prime m-small knots in $S^{3}$. Then $t\left(K_{1} \# K_{2}\right)=$ $t\left(K_{1}\right)+t\left(K_{2}\right)+1$ if and only if neither $K_{1}$ nor $K_{2}$ is $\mu$-primitive.

Kobayashi-Rieck ([3]) further generalized Theorem 1.2 to $m$-small knots in general 3-manifolds. However, Kobayashi-Rieck (4,5]) recently showed that in $S^{3}$ there exist counterexamples to the above conjecture. Note that their counterexamples are non-prime knots, and tunnel number one knots in $S^{3}$ are prime. Moriah (8]) proposed that Conjecture 1.1 be changed to:

Conjecture 1.4 (Morimoto Conjecture). Let $K_{1}, K_{2}$ be two prime knots in $S^{3}$. Then $t\left(K_{1} \# K_{2}\right)=t\left(K_{1}\right)+t\left(K_{2}\right)+1$ if and only if neither $K_{1}$ nor $K_{2}$ is $\mu$-primitive.

In the present paper, we show that Conjecture 1.4 is true for knots $K_{1}, K_{2}$ in which each $K_{i}$ is either tunnel number one or $m$-small, $i=1,2$ :

Theorem 1.5. Let $K_{1}, K_{2}$ be two knots in $S^{3}$, each of which is either tunnel number one or $m$-small. Then $t\left(K_{1} \# K_{2}\right)=t\left(K_{1}\right)+t\left(K_{2}\right)+1$ if and only if neither $K_{1}$ nor $K_{2}$ is $\mu$-primitive.

Clearly, Theorem 1.2 and Theorem 1.3 are direct consequences of Theorem 1.5. In fact, Theorem 1.5 can be obtained from the following theorem.

Theorem 1.6. Let $K_{1}, K_{2}$ be two knots in $S^{3}$, each of which is either tunnel number one or $m$-small. Then $t\left(K_{1} \# K_{2}\right) \geq t\left(K_{1}\right)+t\left(K_{2}\right)$, with equality if and only if either $K_{1}$ or $K_{2}$ is $\mu$-primitive.

The paper is organized as follows. In section 2, we first give some necessary preliminaries and review some known facts; then we prove a lemma which will be used in our proof of the main result. In section 3, we prove Theorem 1.6. 


\section{Preliminaries}

Let $F$ be either a properly embedded connected surface in a 3-manifold $M$ or a sub-surface of $\partial M$. We say $F$ is compressible in $M$ if there is an essential curve in $F$ which bounds a disk in $M$ or if $F$ is a 2-sphere which bounds a 3-ball in $M$. Otherwise, $F$ is incompressible in $M . F$ is bicompressible in $M$ if $F$ is separating and $F$ is compressible in the two complementary components. $M$ is said to be $\partial$-irreducible if $\partial M$ is incompressible. $F$ is an essential surface in $M$ if $F$ is an incompressible surface in $M$ and not parallel to a sub-surface of $\partial M$.

A compression body is a 3 -manifold $V$ obtained from a connected closed orientable surface $S$ by attaching some 2-handles to $S \times\{0\} \subset S \times I$ and capping off any resulting 2-sphere boundary components. We denote $S \times\{1\}$ by $\partial_{+} V$ and $\partial V-\partial_{+} V$ by $\partial_{-} V$. An essential disk for $V$ is a compressing disk of $\partial_{+} V$ in $V$. An incompressible annulus properly embedded in a compression body $V$ is called spanning if one of its two boundary components is in $\partial_{-} V$ and the other is in $\partial_{+} V$.

A Heegaard splitting of a 3-manifold $M$ is a decomposition $V \cup_{S} W$ of $M$ in which $V$ and $W$ are compression bodies such that $V \cap W=\partial_{+} V=\partial_{+} W=S$ and $M=V \cup W . S$ is called a Heegaard surface in $M$. The genus $g(S)$ of $S$ is called the genus of the splitting $V \cup_{S} W$. We use $g(M)$ to denote the Heegaard genus of $M$, which is equal to the minimal genus of all Heegaard splittings of $M$. A Heegaard splitting $V \cup_{S} W$ for $M$ is minimal if $g(S)=g(M)$. A Heegaard splitting $V \cup_{S} W$ is reducible (weakly reducible, or stabilized, respectively) if there are essential disks $D_{1} \subset V$ and $D_{2} \subset W$ such that $\partial D_{1}=\partial D_{2}\left(\partial D_{1} \cap \partial D_{2}=\emptyset\right.$ or $\left|\partial D_{1} \cap \partial D_{2}\right|=1$, respectively). Otherwise, $V \cup_{S} W$ is irreducible (strongly irreducible, unstabilized, respectively). It is a well-known theorem (called Haken's Lemma; see [1]) that any Heegaard splitting of a reducible 3 -manifold is reducible.

It was shown by Scharlemann and Thompson [18] that any irreducible and $\partial-$ irreducible Heegaard splitting $M=V \cup_{S} W$ can be broken up into a series of strongly irreducible Heegaard splittings by rearranging the order of adding the 1and 2-handles as

$$
M=V \cup_{S} W=\left(V_{1} \cup_{S_{1}} W_{1}\right) \cup_{F_{1}}\left(V_{2} \cup_{S_{2}} W_{2}\right) \cup_{F_{2}} \cdots \cup_{F_{m-1}}\left(V_{m} \cup_{S_{m}} W_{m}\right)
$$

such that each $V_{i} \cup_{S_{i}} W_{i}$ is a strongly irreducible Heegaard splitting with $\partial_{-} W_{i}=$ $F_{i}=\partial_{-} V_{i+1}, 1 \leq i \leq m-1, \partial_{-} V_{1}=\partial_{-} V, \partial_{-} W_{m}=\partial_{-} W$, each component of $F_{i}$ is a closed incompressible surface of positive genus, only one component of $M_{i}=V_{i} \cup_{S_{i}} W_{i}$ is not a product, and none of the compression body $V_{i}, W_{i-1}$, $2 \leq i \leq m$, is trivial. Such a rearrangement of handles is called an untelescoping of the Heegaard splitting $V \cup_{S} W$. It is easy to see that $\chi(S) \leq \chi\left(S_{i}\right)-2 \leq \chi\left(F_{i}\right)-4$ for each $i$. There is a natural way to get a Heegaard splitting for $M$ from an untelescoping which is called an amalgamation of the generalized Heegaard splitting ([16]).

Lemma 2.1 ([10]). Let $V$ be a non-trivial compression body and $\mathcal{A}$ be a collection of essential annuli properly embedded in $V$. Then there is an essential disk $D$ in $V$ with $D \cap \mathcal{A}=\emptyset$.

The following lemma is a well-known fact (see [17]).

Lemma 2.2. Let $F$ be an incompressible surface properly embedded in a compression body $V$. Then $\overline{V-F}$ consists of compression bodies. 
Lemma 2.3 ([20]). Let $V$ be a non-trivial compression body and $\mathcal{A}$ be a collection of essential annuli properly embedded in $V$. If $U$ is a component of $\overline{V-\mathcal{A}}$ with $U \cap \partial_{-} V \neq \emptyset$, then $\chi\left(U \cap \partial_{-} V\right) \geq \chi\left(U \cap \partial_{+} V\right)$.

Lemma 2.4 ([17]). Let $F$ be an incompressible surface (not a 2 -sphere, a 2 -disk or a projective plane space) properly embedded in $M=V \cup_{S} W$. If the Heegaard splitting $V \cup_{S} W$ is strongly irreducible, then $F$ can be isotoped so that any component of $S \cap F$ is essential in both $F$ and $S$.

Lemma 2.5 ([19]). Let $M=V \cup_{S} W$ be a strongly irreducible Heegaard splitting. If $\alpha$ is an essential simple loop in $S$ which bounds a disk $D$ in $M$ such that $D$ is transverse to $S$, then $\alpha$ bounds an essential disk in $V$ or $W$.

The next lemma plays an essential role in the proof of the main result. Suppose the knot $K$ is non-trivial in $S^{3}$ in the following.

Lemma 2.6. Suppose $Q$ is a connected bicompressible meridional surface in $E(K)$, and $Q$ cuts $E(K)$ into two compression bodies $N_{1}$ and $N_{2}$. If $Q$ can be compressed into $Q_{i}$ in $N_{i}$ such that any component of $Q_{i}$ is $\partial$-parallel in $E(K)$ for $i=1,2$, then $E(K)$ has a primitive Heegaard splitting $V \cup_{S} W$ with $g(S)=1-\frac{1}{2} \chi(Q)$.

Proof. Since $Q_{1}$ and $Q_{2}$ are meridional surfaces in $E(K)$, there are no disk components in $Q_{1}$ and $Q_{2}$. By assumption, each component of $Q_{1}$ and $Q_{2}$ is a $\partial$-parallel meridional annulus in $E(K)$ and $\left|Q_{1}\right|=\left|Q_{2}\right|$ (where $|\cdot|$ denotes the number of its component). Suppose $\mathcal{D}_{i}$ is a collection of pairwise disjoint compressing disks in $N_{i}$, by which $Q$ is compressed into $Q_{i}$ and $N_{i}$ is cut into $N_{i}^{*}, i=1,2$. Let $A_{i}^{1}, \cdots, A_{i}^{n}$ be all the components of $Q_{i}$, and let $B_{i}^{j}$ be the subsurface of $\partial E(K)$ such that $B_{i}^{j}$ is parallel to $A_{i}^{j}$ and $\partial B_{i}^{j}=\partial A_{i}^{j}$, where $n=\left|Q_{1}\right|, j=1,2, \cdots, n, i=1,2$. We divide it into two cases to discuss:

Case $1(n \geq 2)$. Suppose there exist two different components $B_{1}^{i_{0}}$ and $B_{1}^{j_{0}}$ with $B_{1}^{i_{0}} \subset B_{1}^{j_{0}}$. Set $\mathcal{A}_{1}=\left\{A_{1}^{j}: B_{1}^{j} \subset B_{1}^{j_{0}}, 1 \leq j \leq n, j \neq j_{0}\right\}$ and $\mathcal{A}_{2}=Q_{1}-\left(\mathcal{A}_{1} \cup\right.$ $\left.\left\{A_{1}^{j_{0}}\right\}\right)$. We claim that $\mathcal{A}_{2}=\emptyset$. Otherwise, since $Q$ is connected, there must exist $A_{1}^{i_{1}} \in \mathcal{A}_{1}, A_{1}^{i_{2}} \in \mathcal{A}_{2}$, and $D_{1}, D_{2} \in \mathcal{D}_{1}$ such that the two copies (obtained by compressing $Q$ along $\mathcal{D}_{1}$ ) of $D_{k}$ lie in $A_{1}^{i_{k}}$ and $A_{1}^{j_{0}}$, respectively, $k=1,2$. See Figure 1. This contradicts the assumption that $Q$ is separating in $E(K)$. Thus $\mathcal{A}_{2}=\emptyset$. Let $N$ be the component of $N_{1}^{*}$ which contains $A_{1}^{j_{0}}$; then $N$ is a compression body and $A_{1}^{j_{0}} \subset \partial_{+} N$. Hence $E(K)$ is homeomorphic to a compression body $N$, a contradiction.
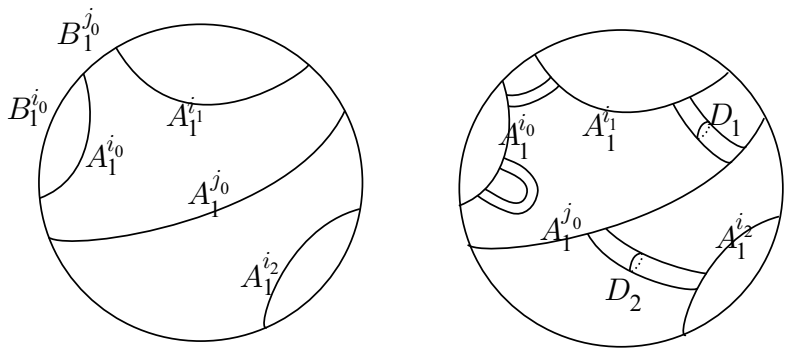

FiguRE 1 
Next assume that no components $A_{1}^{i}, A_{1}^{j}$ of $Q_{1}$ are nested, i.e. $B_{1}^{i} \cap B_{1}^{j}=\emptyset$. Then $N_{1}^{*}=\bigcup_{j=1}^{n} B_{1}^{j} \times I$, where $B_{1}^{j} \times 0=B_{1}^{j}, j=1,2, \cdots, n$. By the same reasons, no two components of $Q_{2}$ are nested and $N_{2}^{*}=\bigcup_{j=1}^{n} B_{2}^{j} \times I$, where $B_{2}^{j} \times 0=B_{2}^{j}$, $j=1,2, \cdots, n$. Set $V=N_{1} \cup\left(\bigcup_{j=1}^{n} B_{2}^{j} \times\left[0, \frac{1}{2}\right]\right)$ and $W=\overline{E(K)-V}$. Then $V$ is obtained from $\partial E(K) \times I$ by adding 1-handles whose co-cores are $\mathcal{D}_{1}$ and $V$ is a compression body. Note that $W=\overline{N_{2}-\bigcup_{i=1}^{r} B_{2}^{i} \times I} \cong N_{2}$ and $W$ is a compression body. Let $S=\partial_{+} V$; then $S=\partial_{+} W$. Thus, $S$ is a Heegaard surface of $E(K)$. Since $\partial E(K) \cap N_{2}$ are annuli, $2-\chi(S)=2-\chi\left(\partial E(K) \cap N_{2}\right)-\chi(Q)=2-\chi(Q)$, i.e. $g(S)=\frac{1}{2}(2-\chi(S))=1-\frac{1}{2} \chi(Q)$. Let $c$ be a component of $\partial B_{2}^{1}$; then $c \times I$ is a spanning annulus in $V$. Let $\alpha$ be an essential arc in $B_{2}^{1} \times \frac{1}{2}$; then there exists an arc $\beta \subset A_{2}^{1}$ with $\beta \subset Q$ and $\partial \alpha=\partial \beta$, which is parallel to $\alpha$ in $N_{2}$. Then $\alpha$ and $\beta$ bound an essential disk $E$ in $W$ with $|\partial E \cap(c \times 1)|=1$. Hence $c \times 1$ is longitude in $W$, which means that the Heegaard splitting $V \cup_{S} W$ is a primitive.

Case $2(n=1)$. If $Q_{1}$ is not $\partial$-parallel in $N_{1}$, then $E(K)$ is homeomorphic to the compression body $N_{1}^{*}$, a contradiction to the assumption. So $Q_{1}$ is $\partial$-parallel in $N_{1}$ and $Q_{2}$ is $\partial$-parallel in $N_{2}$ by the same reason. By the same arguments as in Case 1, there is a primitive Heegaard splitting $V \cup_{S} W$ with $g(S)=1-\frac{1}{2} \chi(Q)$.

\section{The PRoof of Theorem 1.6}

Let $K_{1}, K_{2}$ be two knots in $S^{3}$, and let $K=K_{1} \# K_{2}$. Let $A$ be the decomposing annulus in $E(K)$ which splits $E(K)$ into $E\left(K_{1}\right)$ and $E\left(K_{2}\right)$. Let $A^{\prime}$ be a properly embedded incompressible annulus in $E\left(K_{1}\right)$ with $\partial A^{\prime} \subset A$. Then $\partial A^{\prime}$ divides $\partial E\left(K_{1}\right)$ into two annuli, $A_{1}$ and $A_{2}$. Say $A_{1} \subset A$. We say that $A^{\prime}$ is narrow (respectively, wide) in $E\left(K_{1}\right)$ if $A^{\prime}$ is parallel to $A_{1}$ (respectively, $A_{2}$ ) in $E\left(K_{1}\right)$.

Proof of Theorem 1.6. Let $V \cup_{S} W$ be a minimal genus Heegaard splitting of $E(K)$ with $\partial_{-} V=\partial E(K)$. Obviously $A$ is essential in $E(K)$ and $g(S)=t(K)+1$. We divide it into two cases:

Case 1. The Heegaard splitting $V \cup_{S} W$ is strongly irreducible.

In this case, by Lemma 2.4, we may assume that $S \cap A$ consists of essential loops in both $S$ and $A$ and that $|S \cap A|$ is minimal. Let $S_{1}=S \cap E\left(K_{1}\right), S_{2}=S \cap E\left(K_{2}\right)$. Then each component of $S_{i}$ is a meridional surface in $E\left(K_{i}\right)$, none of which is a disk. $A \cap V$ (respectively, $A \cap W$ ) consists of essential annuli in $V$ (respectively, $W$ ). Let $C_{1}$ (respectively, $C_{2}$ ) be the manifolds obtained by cutting $V$ (respectively, $W$ ) open along $V \cap A$ (respectively, $W \cap A$ ). By Lemma 2.2. each component of $C_{1}$ and $C_{2}$ is a compression body.

By Lemma 2.1, $S_{1}$ or $S_{2}$ is compressible in both $V$ and $W$. Since $V \cup_{S} W$ is strongly irreducible, only one component of $S_{1}$ or $S_{2}$, say $P$, is compressible in both $V$ and $W$, and all the other components are incompressible in both $V$ and $W$. We may assume that $P \subset S_{1}$. For $i=1,2$, let $U_{i}$ be the component of $C_{i}$ which contains $P$; then $P$ is compressible in both $U_{1}$ and $U_{2}$. By maximally compressing $P$ in $U_{i}$, we obtain the surface, denoted by $P_{i}$, each component of which is a meridional surface (not a disk) in $E\left(K_{1}\right)$. Since $V \cup_{S} W$ is strongly irreducible, every component of $P_{i}$ is incompressible in $E\left(K_{1}\right)$ by Lemma 2.5. Then the following two claims are true:

Claim 1. $\chi\left(S_{1}\right) \leq-2 t\left(K_{1}\right)$. Moreover, if $\chi\left(S_{1}\right)=-2 t\left(K_{1}\right)$, then the knot $K_{1}$ is $\mu$-primitive. 
If $\chi(P) \geq-1$, then $P$ is either a disk, an annulus, a twice-punctured disk, or a once-punctured torus, which implies that a component of $\partial P$ bounds a disk in $E\left(K_{1}\right)$ and $A$ is compressible in $E\left(K_{1}\right)$, a contradiction. So $\chi(P) \leq-2$. If one component of $S_{1}$ is essential in $E\left(K_{1}\right)$, then $t\left(K_{1}\right)=1$ and $\chi\left(S_{1}\right)<\chi(P) \leq$ $-2=-2 t\left(K_{1}\right)$; Claim 1 is true. If one component of $P_{1}$ or $P_{2}$ is essential in $E\left(K_{1}\right)$, then by assumption $t\left(K_{1}\right)=1$ and $\chi\left(S_{1}\right) \leq \chi(P)<-2=-2 t\left(K_{1}\right)$; Claim 1 is true again. Thus we may assume that all incompressible components of $S_{1}, P_{1}$ and $P_{2}$ are boundary-parallel annuli in $E\left(K_{1}\right)$. It is easy to see that $U_{1} \cup_{P} U_{2} \cong E\left(K_{1}\right)$. Then by Lemma 2.6 $E\left(K_{1}\right)$ has a primitive Heegaard splitting $V^{\prime} \cup_{S^{\prime}} W^{\prime}$ with $g\left(S^{\prime}\right)=1-\frac{1}{2} \chi(P)$. So $1+t\left(K_{1}\right) \leq g\left(S^{\prime}\right)=1-\frac{1}{2} \chi(P)=1-\frac{1}{2} \chi\left(S_{1}\right)$ and $\chi\left(S_{1}\right) \leq-2 t\left(K_{1}\right)$. Moreover, if $\chi\left(S_{1}\right)=-2 t\left(K_{1}\right)$, then $V^{\prime} \cup_{S^{\prime}} W^{\prime}$ is a minimal Heegaard splitting of $E\left(K_{1}\right)$ and $K_{1}$ is $\mu$-primitive. Hence Claim 1 is true.

Claim 2. $\chi\left(S_{2}\right) \leq-2 t\left(K_{2}\right)$.

Assume that every component of $S_{2}$ is a boundary-parallel annulus in $E\left(K_{2}\right)$. Note that the manifolds obtained by cutting $E\left(K_{2}\right)$ open along $S_{2}$ consist of compression bodies. Precisely, they are solid tori, since each positive boundary is a torus and the negative boundary is empty. Therefore, $E\left(K_{2}\right)$ is a solid torus and $K_{2}$ is a trivial knot, a contradiction. So at least one component of $S_{2}$ is essential in $E\left(K_{2}\right)$, the essential component is not an annulus and $t\left(K_{2}\right)=1$ by assumption. Since any closed surface in $S^{3}$ is separating, $\chi\left(S_{2}\right)$ is even and $\chi\left(S_{2}\right) \leq-2 \leq-2 t\left(K_{2}\right)$. Hence Claim 2 is true.

By Claim 1 and Claim 2, $2 t\left(K_{1} \# K_{2}\right)=-\chi(S)=-\chi\left(S_{1}\right)-\chi\left(S_{2}\right) \geq 2 t\left(K_{1}\right)+$ $2 t\left(K_{2}\right)$, i.e. $t\left(K_{1} \# K_{2}\right) \geq t\left(K_{1}\right)+t\left(K_{2}\right)$, and if $t\left(K_{1} \# K_{2}\right)=t\left(K_{1}\right)+t\left(K_{2}\right)$, then $\chi\left(S_{1}\right)=-2 t\left(K_{1}\right)$ and $K_{1}$ is $\mu$-primitive. We complete the proof of Case 1 .

Case 2. The Heegaard splitting $V \cup_{S} W$ is weakly reducible.

Since $E(K)$ is irreducible, by Haken's lemma, $V \cup_{S} W$ is irreducible. Then by Scharlemann-Thompson's theorem in [18, there exists an untelescoping

$$
\left(V_{1}^{\prime} \cup_{S_{1}^{\prime}} W_{1}^{\prime}\right) \cup_{F_{1}^{\prime}}\left(V_{2}^{\prime} \cup_{S_{2}^{\prime}} W_{2}^{\prime}\right) \cup_{F_{2}^{\prime}} \cdots \cup_{F_{m-1}^{\prime}}\left(V_{m}^{\prime} \cup_{S_{m}^{\prime}} W_{m}^{\prime}\right)
$$

of $V \cup_{S} W$ such that each $V_{i}^{\prime} \cup_{S_{i}^{\prime}} W_{i}^{\prime}(1 \leq i \leq m)$ is a strongly irreducible Heegaard splitting and each $F_{i}^{\prime}(1 \leq i \leq m-1)$ is incompressible in $E(K)$. Let $\mathcal{F}=\bigcup_{i=1}^{m-1} F_{i}^{\prime}$ and $\mathcal{S}=\bigcup_{i=1}^{m} S_{i}^{\prime}$. Since both $\mathcal{F}$ and $A$ are incompressible in $E(K)$, we may assume that $\mathcal{F} \cap A$ consists of essential loops in both $\mathcal{F}$ and $A$. Since each $V_{i}^{\prime} \cup_{S_{i}^{\prime}} W_{i}^{\prime}$ is strongly irreducible, by Lemma 2.4 we may further assume $\mathcal{S} \cap A$ consists of essential loops in both $\mathcal{S}$ and $A$, and $|A \cap(\mathcal{F} \cup \mathcal{S})|$ is minimal. Let $V_{j}^{\prime} \cup_{S_{j}^{\prime}} W_{j}^{\prime}$ be the innermost component among all $V_{i}^{\prime} \cup_{S_{i}^{\prime}} W_{i}^{\prime}$ with $S_{i}^{\prime} \cap A \neq \emptyset$, which means that $S_{j}^{\prime}$ bounds a 3-manifold $M^{\prime}$ with $\partial E(K) \cap M^{\prime}=\emptyset$ and $\left(\mathcal{S} \cap M^{\prime}\right) \cap A=S_{j}^{\prime} \cap A \neq \emptyset$. Set $S_{i}^{0}=S_{j}^{\prime} \cap E\left(K_{i}\right)$ for $i=1,2$. Then by Lemma 2.3, for $i=1,2$, the following inequality holds:

$$
\begin{aligned}
& \chi\left(\mathcal{S} \cap E\left(K_{i}\right)\right)-\chi\left(\mathcal{F} \cap E\left(K_{i}\right)\right) \\
= & \Sigma_{k=1}^{j-1}\left(\chi\left(S_{k}^{\prime} \cap E\left(K_{i}\right)\right)-\chi\left(F_{k}^{\prime} \cap E\left(K_{i}\right)\right)\right) \\
& +\Sigma_{k=j}^{m-1}\left(\chi\left(S_{k+1}^{\prime} \cap E\left(K_{i}\right)\right)-\chi\left(F_{k}^{\prime} \cap E\left(K_{i}\right)\right)\right)+\chi\left(S_{j}^{\prime} \cap E\left(K_{i}\right)\right) \\
\leq & \chi\left(S_{j}^{\prime} \cap E\left(K_{i}\right)\right) .
\end{aligned}
$$


By Lemma 2.1, $S_{1}^{0}$ or $S_{2}^{0}$ is compressible in both $V_{j}^{\prime}$ and $W_{j}^{\prime}$. Since $V_{j}^{\prime} \cup_{S_{j}^{\prime}} W_{j}^{\prime}$ is strongly irreducible, only one component, say $P^{0}$, of $S_{1}^{0}$ or $S_{2}^{0}$ is compressible in both $V_{j}^{\prime}$ and $W_{j}^{\prime}$, and all the other components are incompressible in both $V_{j}^{\prime}$ and $W_{j}^{\prime}$. We may assume that $P^{0} \subset S_{1}^{0}$. By Lemma 2.2, any component of $\overline{E(K)-(\mathcal{S} \cup \mathcal{F} \cup A)}$ is a compression body. Then we have the following two claims:

Claim 3. $\chi\left(\mathcal{S} \cap E\left(K_{2}\right)\right)-\chi\left(\mathcal{F} \cap E\left(K_{2}\right)\right) \leq-2 t\left(K_{2}\right)$.

If one component of $S_{2}^{0}$ is essential in $E\left(K_{2}\right)$, then by assumption $t\left(K_{2}\right)=$ 1. Since any closed surface in $S^{3}$ is separating, $\chi\left(S_{2}^{0}\right)$ is even, and if there is a component of $S_{2}^{0}$ which is not an annulus, then by the inequality $(*), \chi(\mathcal{S} \cap$ $\left.E\left(K_{2}\right)\right)-\chi\left(\mathcal{F} \cap E\left(K_{2}\right)\right) \leq \chi\left(S_{j}^{\prime} \cap E\left(K_{2}\right)\right)=\chi\left(S_{2}^{0}\right) \leq-2=-2 t\left(K_{2}\right)$. If any component of $S_{2}^{0}$ is a boundary-parallel annulus in $E\left(K_{2}\right), S_{2}^{0}$ must be nested wide annuli in $E\left(K_{2}\right)$ since $|A \cap(\mathcal{F} \cup \mathcal{S})|$ is minimal. Let $B$ be the innermost wide annulus among $S_{2}^{0}$; then $\partial B$ bounds an annulus, denoted by $A^{*}$, in $A$. Since $V_{j}^{\prime} \cup_{S_{j}^{\prime}} W_{j}^{\prime}$ and $B$ are innermost, $B$ and $A^{*}$ bound a compression body, denoted by $C$, of $\overline{E(K)-(\mathcal{S} \cup \mathcal{F} \cup A)}$ with $\partial_{+} C=B \cup A^{*}$. If $\partial_{-} C=\emptyset$, then $E\left(K_{2}\right) \cong C$ is a solid torus and $K_{2}$ is a trivial knot, a contradiction. Hence $\partial_{-} C \neq \emptyset, C$ is a torus $\times I$ and $\partial_{-} C$ is boundary parallel in $E\left(K_{2}\right)$. Note that $\partial_{-} C \subset \mathcal{F}, \partial_{-} C$ bounds a subuntelescoping of $\left(V_{1}^{\prime} \cup_{S_{1}^{\prime}} W_{1}^{\prime}\right) \cup_{F_{1}^{\prime}}\left(V_{2}^{\prime} \cup_{S_{2}^{\prime}} W_{2}^{\prime}\right) \cup_{F_{2}^{\prime}} \cdots \cup_{F_{m-1}^{\prime}}\left(V_{m}^{\prime} \cup_{S_{m}^{\prime}} W_{m}^{\prime}\right)$, denoted by $\left(V_{k+1}^{*} \cup_{S_{k+1}^{*}} W_{k+1}^{*}\right) \cup_{F_{k+1}^{*}}\left(V_{k+2}^{*} \cup_{S_{k+2}^{*}} W_{k+2}^{*}\right) \cup_{F_{k+2}^{*}} \cdots \cup_{F_{m-1}^{*}}\left(V_{m}^{*} \cup_{S_{m}^{*}} W_{m}^{*}\right)$, which can be amalgamated to a Heegaard splitting $V^{*} \cup_{S^{*}} W^{*}$ of $E\left(K_{2}\right)$, where $V_{i}^{*} \cup_{S_{i}^{*}} W_{i}^{*}$ is a component of $V_{i}^{\prime} \cup_{S_{i}^{\prime}} W_{i}^{\prime}$ for $k+1 \leq i \leq m$. After the amalgamation, we have $\chi\left(\mathcal{S} \cap E\left(K_{2}\right)\right)-\chi\left(\mathcal{F} \cap E\left(K_{2}\right)\right)=\chi\left(S^{*}\right) \leq-2 t\left(K_{2}\right)$. Hence Claim 3 is true.

Claim 4. $\chi\left(\mathcal{S} \cap E\left(K_{1}\right)\right)-\chi\left(\mathcal{F} \cap E\left(K_{1}\right)\right) \leq-2 t\left(K_{1}\right)$. Moreover, if $\chi\left(\mathcal{S} \cap E\left(K_{1}\right)\right)-$ $\chi\left(\mathcal{F} \cap E\left(K_{1}\right)\right)=-2 t\left(K_{1}\right)$, then $K_{1}$ is $\mu$-primitive.

For $i=1,2$, let $U_{i}^{0}$ be the component of $\overline{E(K)-(\mathcal{S} \cup \mathcal{F} \cup A)}$ which contains $P^{0}$. Then $P^{0}$ is compressible in both $U_{1}^{0}$ and $U_{2}^{0}$, and by maximally compressing $P^{0}$ in $U_{i}^{0}$, we obtain a surface, denoted by $P_{i}^{0}$, each component of which is a meridional surface (not a disk) in $E\left(K_{1}\right)$. Since $V_{j}^{\prime} \cup_{S_{j}^{\prime}} W_{j}^{\prime}$ is strongly irreducible, any component of $P_{1}^{0}$ and $P_{2}^{0}$ is incompressible in $E\left(K_{1}\right)$ by Lemma 2.5. If one component of $S_{1}^{0}, P_{1}^{0}$ or $P_{2}^{0}$, say $P^{*}$, is essential in $E\left(K_{1}\right)$, then $t\left(K_{1}\right)=1$. By the inequality $(*), \chi\left(\mathcal{S} \cap E\left(K_{1}\right)\right)-\chi\left(\mathcal{F} \cap E\left(K_{1}\right)\right) \leq \chi\left(S_{j}^{\prime} \cap E\left(K_{1}\right)\right) \leq \chi\left(S_{1}^{0}\right) \leq$ $\chi\left(P^{*}\right)-2<-2=-2 t\left(K_{1}\right)$, and Claim 4 is true. So we may assume that any incompressible component of $S_{1}^{0}$ is a boundary-parallel annulus in $E\left(K_{1}\right)$, and any component with boundary of both $P_{1}^{0}$ and $P_{2}^{0}$ is a boundary-parallel annulus in $E\left(K_{1}\right)$.

If both $P_{1}^{0}$ and $P_{2}^{0}$ have no closed surface component, then $U_{1}^{0} \cup_{P^{0}} U_{2}^{0} \cong E\left(K_{1}\right)$ and $P^{0}$ satisfies the conditions of Lemma 2.6 in $E\left(K_{1}\right)$. By Lemma 2.6] $E\left(K_{1}\right)$ has a primitive Heegaard splitting $V^{0} \cup_{S^{0}} W^{0}$ with $g\left(S^{0}\right)=1-\frac{1}{2} \chi\left(P^{0}\right)$. So $1+t\left(K_{1}\right) \leq$ $g\left(S^{0}\right)=1-\frac{1}{2} \chi\left(P^{0}\right)=1-\frac{1}{2} \chi\left(S_{1}^{0}\right)$ and $\chi\left(S_{1}^{0}\right) \leq-2 t\left(K_{1}\right)$. By the inequality $(*)$, $\chi\left(\mathcal{S} \cap E\left(K_{1}\right)\right)-\chi\left(\mathcal{F} \cap E\left(K_{1}\right)\right) \leq \chi\left(S_{j}^{\prime} \cap E\left(K_{1}\right)\right) \leq \chi\left(S_{1}^{0}\right) \leq-2 t\left(K_{1}\right)$, and if $\chi\left(\mathcal{S} \cap E\left(K_{1}\right)\right)-\chi\left(\mathcal{F} \cap E\left(K_{1}\right)\right)=-2 t\left(K_{1}\right)$, then $V^{0} \cup_{S^{0}} W^{0}$ is a minimal Heegaard splitting of $E\left(K_{1}\right)$ and $K_{1}$ is $\mu$-primitive, and Claim 4 is true. 
If $P_{1}^{0}$ or $P_{2}^{0}$ has closed surface components, then those closed components must be parallel to $\mathcal{F}$ since a closed incompressible surface in a compression body is $\partial$-parallel in the compression body. Let $\mathcal{F}_{1}$ be the components of $\mathcal{F}$ which are contained in $E\left(K_{1}\right)$. $\mathcal{F}_{1}$ bounds a sub-untelescoping of the foregoing untelescoping. By the amalgamation of the sub-untelescoping and $V_{j}^{\prime} \cup_{S_{j}^{\prime}} W_{j}^{\prime}$ along $\mathcal{F}_{1}$, denote the resulting Heegaard splitting by $V_{j}^{\prime \prime} \cup_{S_{j}^{\prime \prime}} W_{j}^{\prime \prime}$. By the amalgamation, we can assume that $S_{j}^{\prime} \cap E\left(K_{2}\right)=S_{j}^{\prime \prime} \cap E\left(K_{2}\right)$, the incompressible components of $S_{j}^{\prime} \cap E\left(K_{1}\right)$ are the same as the incompressible components $S_{j}^{\prime \prime} \cap E\left(K_{1}\right)$ and only one component, say $P^{*}$, of $S_{j}^{\prime \prime} \cap E\left(K_{1}\right)$ is compressible in both $V_{j}^{\prime \prime}$ and $W_{j}^{\prime \prime}$.

For $i=1,2$, set $S_{i}^{*}=S_{j}^{\prime \prime} \cap E\left(K_{i}\right)$. Denote $P_{1}^{*}\left(P_{2}^{*}\right.$, respectively) as the resulting components by maximally compressing $P^{*}$ in $V_{j}^{\prime \prime}\left(W_{j}^{\prime \prime}\right.$, respectively). Then we may assume that any incompressible component of $S_{j}^{\prime \prime} \cap E\left(K_{1}\right)$ and any component of both $P_{1}^{*}$ and $P_{2}^{*}$ are boundary-parallel annuli in $E\left(K_{1}\right)$. Hence $P^{*}$ satisfies the conditions of Lemma 2.6 in $E\left(K_{1}\right)$. Then by Lemma 2.6 $E\left(K_{1}\right)$ has a primitive Heegaard splitting $V^{0} \cup_{S^{0}} W^{0}$ with $g\left(S^{0}\right)=1-\frac{1}{2} \chi\left(P^{*}\right)$. So $1+t\left(K_{1}\right) \leq g\left(S^{0}\right)=$ $1-\frac{1}{2} \chi\left(P^{*}\right)=1-\frac{1}{2} \chi\left(S_{1}^{*}\right)$ and $\chi\left(S_{1}^{*}\right) \leq-2 t\left(K_{1}\right)$. By the inequality $(*), \chi(\mathcal{S} \cap$ $\left.E\left(K_{1}\right)\right)-\chi\left(\mathcal{F} \cap E\left(K_{1}\right)\right) \leq \chi\left(S_{j}^{\prime \prime} \cap E\left(K_{1}\right)\right) \leq \chi\left(S_{1}^{*}\right) \leq-2 t\left(K_{1}\right)$, and if $\chi(\mathcal{S} \cap$ $\left.E\left(K_{1}\right)\right)-\chi\left(\mathcal{F} \cap E\left(K_{1}\right)\right)=-2 t\left(K_{1}\right)$, then $V^{0} \cup_{S^{0}} W^{0}$ is a minimal Heegaard splitting of $E\left(K_{1}\right)$ and $K_{1}$ is $\mu$-primitive. Hence Claim 4 is true.

By Claim 3 and Claim 4, $-2 t\left(K_{1} \# K_{2}\right)=\chi(S)=\chi\left(\mathcal{S} \cap E\left(K_{1}\right)\right)-\chi\left(\mathcal{F} \cap E\left(K_{1}\right)\right)+$ $\chi\left(\mathcal{S} \cap E\left(K_{2}\right)\right)-\chi\left(\mathcal{F} \cap E\left(K_{2}\right)\right) \leq-2 t\left(K_{1}\right)-2 t\left(K_{2}\right)$, i.e. $t\left(K_{1} \# K_{2}\right) \geq t\left(K_{1}\right)+$ $t\left(K_{2}\right)$, and if $t\left(K_{1} \# K_{2}\right)=t\left(K_{1}\right)+t\left(K_{2}\right)$, then $\chi\left(\mathcal{S} \cap E\left(K_{1}\right)\right)-\chi\left(\mathcal{F} \cap E\left(K_{1}\right)\right)=$ $-2 t\left(K_{1}\right)$ and $K_{1}$ is $\mu$-primitive. This completes the proof of Case 2 and the proof of Theorem 1.6 .

\section{ACKNOWLEDGEMENTS}

The authors thank the referee for helpful suggestions and useful comments. They also thank Professor Daniel Ruberman for helpful comments.

\section{REFERENCES}

[1] A. J. Casson and C. McA. Gordon, Reducing Heegaard splittings, Topology Appl. 27 (1987), no. 3, 275-283, DOI 10.1016/0166-8641(87)90092-7. MR918537 (89c:57020)

[2] Tsuyoshi Kobayashi, A construction of arbitrarily high degeneration of tunnel numbers of knots under connected sum, J. Knot Theory Ramifications 3 (1994), no. 2, 179-186, DOI 10.1142/S0218216594000137. MR.1279920 (95g:57011)

[3] Tsuyoshi Kobayashi and Yo'av Rieck, Heegaard genus of the connected sum of m-small knots, Comm. Anal. Geom. 14 (2006), no. 5, 1037-1077. MR2287154(2007i:57018)

[4] Tsuyoshi Kobayashi and Yo'av Rieck, Knot exteriors with additive Heegaard genus and Morimoto's conjecture, Algebr. Geom. Topol. 8 (2008), no. 2, 953-969, DOI 10.2140/agt.2008.8.953. MR2443104 (2009m:57011)

[5] Tsuyoshi Kobayashi and Yo'av Rieck, Knots with $g(E(K))=2$ and $g(E(K \# K \# K))=$ 6 and Morimoto's conjecture, Topology Appl. 156 (2009), no. 6, 1114-1117, DOI 10.1016/j.topol.2008.10.003. MR2493371 (2010b:57007)

[6] Martin Lustig and Yoav Moriah, Generalized Montesinos knots, tunnels and $\mathcal{N}$-torsion, Math. Ann. 295 (1993), no. 1, 167-189, DOI 10.1007/BF01444882. MR1198847 (94b:57011)

[7] Martin Lustig and Yoav Moriah, Closed incompressible surfaces in complements of wide knots and links, Topology Appl. 92 (1999), no. 1, 1-13, DOI 10.1016/S0166-8641(97)002320. MR1670164 (2000b:57009) 
[8] Yoav Moriah, Heegaard splittings of knot exteriors, Workshop on Heegaard Splittings, Geom. Topol. Monogr., vol. 12, Geom. Topol. Publ., Coventry, 2007, pp. 191-232, DOI 10.2140/gtm.2007.12.191. MR2408247 (2009i:57015)

[9] Yoav Moriah and Hyam Rubinstein, Heegaard structures of negatively curved 3-manifolds, Comm. Anal. Geom. 5 (1997), no. 3, 375-412. MR.1487722 (98j:57029)

[10] Kanji Morimoto, Tunnel number, connected sum and meridional essential surfaces, Topology 39 (2000), no. 3, 469-485, DOI 10.1016/S0040-9383(98)00070-6. MR1746903 (2001a:57015)

[11] Kanji Morimoto, On the additivity of tunnel number of knots, Topology Appl. 53 (1993), no. 1, 37-66, DOI 10.1016/0166-8641(93)90099-Y. MR.1243869 (94j:57011)

[12] Kanji Morimoto, On the super additivity of tunnel number of knots, Math. Ann. 317 (2000), no. 3, 489-508, DOI 10.1007/PL00004411. MR1776114 (2001g:57016)

[13] Kanji Morimoto, There are knots whose tunnel numbers go down under connected sum, Proc. Amer. Math. Soc. 123 (1995), no. 11, 3527-3532, DOI 10.2307/2161103. MR1317043 (96a:57022)

[14] Kanji Morimoto and Jennifer Schultens, Tunnel numbers of small knots do not go down under connected sum, Proc. Amer. Math. Soc. 128 (2000), no. 1, 269-278, DOI 10.1090/S0002-993999-05160-6. MR.1641065 (2000c:57014)

[15] Kanji Morimoto, Makoto Sakuma, and Yoshiyuki Yokota, Examples of tunnel number one knots which have the property " $1+1=3$ ", Math. Proc. Cambridge Philos. Soc. 119 (1996), no. 1, 113-118, DOI 10.1017/S0305004100074028. MR1356163 (96i:57007)

[16] Jennifer Schultens, The classification of Heegaard splittings for (compact orientable surface) $\times S^{1}$, Proc. London Math. Soc. (3) 67 (1993), no. 2, 425-448, DOI 10.1112/plms/s367.2.425. MR1226608(94d:57043)

[17] Jennifer Schultens, Additivity of tunnel number for small knots, Comment. Math. Helv. 75 (2000), no. 3, 353-367, DOI 10.1007/s000140050131. MR1793793 (2001i:57012)

[18] Martin Scharlemann and Abigail Thompson, Thin position for 3-manifolds, Geometric topology (Haifa, 1992), Contemp. Math., vol. 164, Amer. Math. Soc., Providence, RI, 1994, pp. 231-238, DOI 10.1090/conm/164/01596. MR/1282766 (95e:57032)

[19] Martin Scharlemann, Local detection of strongly irreducible Heegaard splittings, Topology Appl. 90 (1998), no. 1-3, 135-147, DOI 10.1016/S0166-8641(97)00184-3. MR1648310 (99h:57040)

[20] Martin Scharlemann and Jennifer Schultens, The tunnel number of the sum of $n$ knots is at least $n$, Topology 38 (1999), no. 2, 265-270, DOI 10.1016/S0040-9383(98)00002-0. MR.1660345(2000b:57013)

School of Astronautics and Department of Mathematics, Harbin Institute of TechNOlogy, Harbin 150001, People's Republic of China

E-mail address: gqyang@hit.edu.cn

Department of Mathematics, Harbin Institute of Technology, Harbin 150001, PeoPLE'S REPUBLIC OF CHINA

E-mail address: jlxbyin@hit.edu.cn

School of Mathematical Sciences, Dalian University of Technology, Dalian 116024, People's Republic of China

E-mail address: ffcclei@yahoo.com.cn 